\title{
Integral for longitudinal phase space tomography on equilibrium distributions
}

\author{
Leo Michelotti \\ Fermilab, P.O. Box 500, MS220, Batavia, Illinois 60510 \\ (Received 18 November 2002; published 14 February 2003; publisher error corrected 11 March 2003)

\begin{abstract}
We employ Abel's transform to derive an analytic expression for filtering an equilibrium distribution function from its longitudinal beam profile. The result can be applied to a large class of Hamiltonians that are quadratic in the momentum coordinate.
\end{abstract}

DOI: 10.1103/PhysRevSTAB.6.024001

PACS numbers: 41.85.Ew, 45.20.Jj

\section{INTRODUCTION}

Longitudinal phase space tomography is developing rapidly into a practical diagnostic technique. In an early proposal, Jackson [1] suggested using the tomographic Algebraic Reconstruction Technique to image distributions before coalescing in Fermilab's Main Ring. A numerical code written by Hancock, Lindroos, and Koscielniak [2] at CERN has constructed a distribution function from a collection of changing beam profiles using a tracking function that included space charge effects. Montag, D'Imperio, Kewisch, and Lee [3] wrote their own code and obtained remarkable images of an instability that developed during rebucketing at RHIC. Schlarb [4] described a procedure used at DESY, based on a thesis of Geitz [5], for reconstruction of transverse distributions. More recently, Huening [6] constructed longitudinal tomographic images to study wake field effects at the Tesla Test Facility.

When a longitudinal distribution is in equilibrium, one profile should be sufficient to reconstruct it. Tan developed a numerical procedure for doing this when the bucket is stationary (nonaccelerating) [7]. The procedure can be modified to work on nonstationary (accelerating) buckets. In either case one obtains a lower triangular linear system of equations which must be inverted, not an unusual occurrence in tomographic problems.

The purpose of this paper is to present an analytic expression for obtaining an equilibrium distribution function from a beam profile, an integral transform whose numerical implementation is equivalent to inverting that triangular system of equations. In the next section, we go through a simple exercise to set up the solution; in the third, we extend the exercise to longitudinal phase space and, by implication, to a large class of Hamiltonians quadratic in the momentum coordinate. The final section contains a few closing comments.

\section{RADIALLY SYMMETRIC DISTRIBUTIONS: ABELS EQUATION}

We begin by reviewing an exercise so as to reduce notational complexity later. Let $X$ and $Y$ be a pair of real, random variables and suppose that their distribution in the Euclidean plane depends only on radius, say,

$$
\text { Probability }[(X, Y) \in \mathcal{D}]=\int_{\mathcal{D}} d x d y f\left(r^{2}\right),
$$

where $r^{2}=x^{2}+y^{2}$. Consider the projections at fixed values of $x$.

$$
g(x) \equiv \int_{-\infty}^{\infty} d y f\left(r^{2}\right)=2 \int_{0}^{\infty} d y f\left(r^{2}\right) .
$$

Because $d x=0$ along the path of integration,

$$
2 y d y=d\left(r^{2}\right) \Rightarrow 2 d y=\frac{d\left(r^{2}\right)}{\sqrt{r^{2}-x^{2}}}, \quad \text { for } y>0 .
$$

We then can write

$$
g(x)=\int_{x^{2}}^{\infty} d u \frac{f(u)}{\sqrt{u-x^{2}}},
$$

with $u \equiv r^{2}$.

This is much like an integral equation motivated by one of those "bead on a frictionless wire" problems: Suppose that a mass drops in a constant gravitational field along a contrained path, expressed as the set of points $\left\{(y(z), z) \in E^{2} \mid z \in[0, \infty)\right\}$. Let the time that it takes for the bead to reach $z=0$ when it is dropped from an altitude $z=h$ be represented by the function $\tau(h)$. Because $y(z)$ is a function, the path possesses no roller coaster dips or extended regions of zero slope. We therefore can assume that (a) $\tau(h)$ is continuous and (b) $\tau(0)=0$. Under those conditions, is it possible to determine $y(z)$ from $\tau(h)$ ?

The answer, yes, was determined by the Norwegian mathematician Abel [8,9] in 1823 [10], about one year before he proved the impossibility of solving, "in closed form," polynomial equations of degree greater than 4 . The problem is expressed as an integral equation by using conservation of energy:

$$
\frac{1}{2} m v^{2}+m g z=m g h \Rightarrow v=\sqrt{2 g(h-z)}
$$

means that

$$
\tau(h)=\int_{0}^{\tau(h)} d t=\int \frac{d s}{v}=\int_{0}^{h} d z \frac{d s / d z}{\sqrt{2 g(h-z)}},
$$

where $d s$ is differential arc length along the path. Abel showed how to invert this to obtain $d s / d z$, after which the task of determining $y(z)$ is reduced to quadrature. 
A more general form of his problem, and its solutions, can be found in texts on integral equations, such as Hochstadt [11] or Pogorzelski [12]. Let

$$
\psi(t)=\int_{0}^{t} d z \frac{\phi(z)}{(t-z)^{\alpha}},
$$

where (a) $0 \leq \alpha<1$, (b) $t \geq 0$, (c) $\psi(t)$ is continuous, and (d) $\psi(0)=0$. Inversion is accomplished by any of the following expressions:

$$
\begin{aligned}
\phi(z) & =\frac{\sin \pi \alpha}{\pi} \frac{d}{d z} \int_{0}^{z} d t \frac{\psi(t)}{(z-t)^{1-\alpha}} \\
& =\frac{\sin \pi \alpha}{\pi} \int_{t=0}^{t=z} \frac{d \psi(t)}{(z-t)^{1-\alpha}} \\
& =\frac{\sin \pi \alpha}{\pi \alpha}\left(\psi^{\prime}(0) z^{\alpha}+\int_{0}^{z} d t \frac{d^{2} \psi}{d t^{2}}(z-t)^{\alpha}\right) .
\end{aligned}
$$

Equation (6) is obtained from Eq. (5) via an integration by parts. It possesses the advantage of a nonsingular kernel in the integrand and the disadvantage of requiring two derivatives of the "signal" function, $\psi$. This particular expression makes it convenient to verify explicitly that $\varphi(z) \rightarrow \psi^{\prime}(z)$ as $\alpha \rightarrow 0$. In what follows, we will work preferentially with expression (5).

Equation (1) is connected to Eq. (3) by changing the integration coordinate:

$$
u v=1 \Rightarrow d u=-(u / v) d v,
$$

so that, after a little algebra, Eq. (1) is rewritten as follows:

$$
|x| g(x)=\int_{0}^{1 / x^{2}} d v\left(\frac{f(1 / v)}{v^{3 / 2}}\right) \frac{1}{\sqrt{1 / x^{2}-v}} .
$$

Notice that multiplication by $|x|$ suppresses what we expect will be the strongest part of the signal. This is now in the same form as Eq. (3), as can be seen via substitutions [13].

$$
\begin{aligned}
\alpha & \rightarrow 1 / 2, \\
z & \rightarrow v, \\
t & \rightarrow 1 / x^{2}, \\
\phi(z) & \rightarrow \frac{f(1 / v)}{v^{3 / 2},} \\
\psi(t) & \rightarrow|x| g(x) .
\end{aligned}
$$

[If it is helpful, think of $|x| g(x)$ as defining a function $G\left(1 / x^{2}\right)$.] Conditions (a) through (d) are satisfied, so the inversion can be written immediately by reversing these substitutions in Eq. (5).

$$
\frac{f(1 / v)}{v^{3 / 2}}=\frac{1}{\pi} \int_{1 / x^{2}=0}^{1 / x^{2}=v} \frac{d(|x| g(x))}{\sqrt{v-1 / x^{2}}} .
$$

Now write

$$
\begin{aligned}
d(|x| g(x)) & =d\left(1 / x^{2}\right) \frac{d(|x| g(x))}{d\left(1 / x^{2}\right)}=d x \frac{d(|x| g(x))}{d x} \\
& \equiv d x(|x| g(x))^{\prime},
\end{aligned}
$$

so that, upon (arbitrarily) choosing $x>0$,

$$
\frac{f(1 / v)}{v^{3 / 2}}=\frac{1}{\pi} \int_{\infty}^{1 / \sqrt{v}} d x \frac{(x g(x))^{\prime}}{\sqrt{v-1 / x^{2}}} .
$$

As a final step, we go back and use $v=1 / u=1 / r^{2}$ to express this as follows:

$$
r^{2} f\left(r^{2}\right)=\frac{1}{\pi} \int_{\infty}^{r} d x \frac{(x g(x))^{\prime}}{\sqrt{1-(r / x)^{2}}} .
$$

Abel's equation was first applied in this way to tomography by Radon [14], as Cormack, who shared the Nobel Prize in 1979 for inventing the CAT scan, noted in his acceptance speech.

"[T]his seemed like a problem which would have been solved before, probably in the 19th century, but again a literature search and enquiries of mathematicians provided no information about it. Fourteen years would elapse before I learned that Radon had solved this problem in 1917 ... The solution is easy for objects with circular symmetry .... One has Abel's equation to solve, and its solution has been known since 1825. [sic]"

I am indebted to a reviewer for pointing out that Krempl [15] and Kats [16] applied Abel's integral to equilibrium distributions over the phase space of a simple harmonic oscillator. In addition, Carli and Chanel [17] have used it in the context of transverse electron cooling.

\section{EXTENSION TO LONGITUDINAL PHASE SPACE}

We will now extend Eq. (7) to the problem of determining an equilibrium distribution in longitudinal phase space. For convenience, we write the Hamiltonian model (a) using coordinates centered at the synchronous phase and energy, $\varphi_{s}$ and $E_{s}$, and (b) shifted so that the Hamiltonian vanishes at the point of synchrony, $H\left(\varphi_{s}, E_{s}\right)=0$. Because of (a), the kick imparted to a particle in the corresponding map model would change its energy according to

$$
\Delta E=e V \sin \left(\varphi_{s}+\varphi\right),
$$

where $e V>0$ is the maximum possible energy imparted by the cavity. A model satisfying these two conditions is written

$$
\begin{aligned}
H & =-\frac{1}{2} \alpha W^{2}+e V \cdot\left[\cos \left(\varphi_{s}+\varphi\right)-\cos \varphi_{s}+\varphi \sin \varphi_{s}\right] \\
& \equiv-\frac{1}{2} \alpha W^{2}+\mathcal{U}\left(\varphi ; \varphi_{s}\right),
\end{aligned}
$$

where 


$$
\alpha=\frac{h}{2 \pi}\left[\left(\frac{\omega}{p c}\right)^{2} \cdot E \cdot\left(\frac{1}{\gamma^{2}}-\frac{1}{\gamma_{t}^{2}}\right)\right]_{s}
$$

and

$$
W=\left(E-E_{s}\right)\left(2 \pi / \omega_{s}\right)=\left(E-E_{s}\right) \tau_{s} .
$$

The subscript $s$ always stands for "evaluated at the point of synchrony." The "potential" function $\mathcal{U}$ is effectively defined by Eq. (8). $\varphi \in R$ and $W \in R$ are canonical coordinates.

Let $f(\varphi, W)$ be a continuous distribution over this longitudinal phase space, normalized so that

Probability (particle in $\mathcal{D})=\int_{\mathcal{D}} d \varphi d W f(\varphi, W)$.

Without collisions, $f$ satisfies the Vlasov equation,

$$
\{f, H\}+\frac{\partial f}{\partial t}=0 .
$$

If the distribution is in equilibrium, $\partial f / \partial t=0$, so that the Poisson bracket itself vanishes, $\{f, H\}=0$. Our model is two dimensional and integrable. The only functions which "commute" with the Hamiltonian must be constant along its isosurfaces. Thus, for some function, $g: R \rightarrow R$, we can write $f=g \circ H, \quad$ or $f(\varphi, W)=$ $g(H(\varphi, W))$.

A beam profile will be represented as the projection,

$$
\begin{aligned}
S(\varphi) & =\int_{-\infty}^{\infty} d W f(\varphi, W)=\int_{-\infty}^{\infty} d W g(H(\varphi, W)) \\
& =2 \int_{-\infty}^{0} d W g(H(\varphi, W)) .
\end{aligned}
$$

I have used the symmetry of $H$ to reduce the region of integration. We are using $\varphi$ to parametrize the signal. However, it will more likely be recorded as a function of time, $t$, where $\varphi=\omega_{\mathrm{rf}}\left(t-t_{s}\right)$. To make matters worse, shortly we will want to use $\sqrt{\mathcal{U}(\varphi)}$ as the parametrization coordinate. To avoid a confusing jumble of terminology - an all but impossible task, under the circumstances - we introduce notation,

$$
S(\varphi) \equiv S_{\Phi}(\varphi) \equiv S_{T}(t) \equiv S_{U}(\sqrt{\mathcal{U}}),
$$

and anticipate using the appropriate representation as needed. Our objective is to invert Eq. (9) and determine $g$ from $S$ [18].

We have reduced the domain of integration over $W$ so that, given a fixed value of $\varphi$, the reduced maps $W \mapsto H$ and $H \mapsto W$, are one-to-one when restricted to it. Thus, over this domain, $W \in(-\infty, 0), H$ itself can be used as a coordinate. Of course, we could just as well have chosen $W \in(0, \infty)$ and obtained equivalent results. Accordingly, we change the integration coordinate from $W$ to $H$.

$$
S(\varphi)=2 \int_{\Gamma} d H\left(\frac{\partial W}{\partial H}\right)_{\varphi} g(H)=2 \int_{\Gamma} d H\left(-\frac{1}{\alpha W}\right) g(H) .
$$

The range of integration, $\Gamma$, depends on whether the system is above or below transition. It will be written explicitly below. To make clearer that $H$ is used here as an integration coordinate, not as the name of a function, let us introduce a new symbol, $\mathcal{H}$, and write this as

$$
S_{\Phi}(\varphi)=-\frac{2}{\alpha} \int_{\Gamma} d \mathcal{H} \frac{g(\mathcal{H})}{W(\varphi, \mathcal{H})}
$$

Now use Eq. (8) to substitute for $W$.

$$
[\alpha W(\varphi, \mathcal{H})]^{2}=-2 \alpha\left[\mathcal{H}-\mathcal{U}\left(\varphi ; \varphi_{s}\right)\right] .
$$

Over our domain, $W \in(-\infty, 0), W<0$, and

above transition: $\alpha<0$,

$$
\begin{gathered}
1 / \alpha W>0, \text { and } \\
0 \leq \mathcal{U}(\varphi) \leq H(\varphi, W)
\end{gathered}
$$

below transition: $\alpha>0$,

$$
\begin{gathered}
1 / \alpha W<0, \text { and } \\
H(\varphi, W) \leq \mathcal{U}(\varphi) \leq 0,
\end{gathered}
$$

where $\mathcal{U}$ and $H$ are evaluated inside the separatrix. We rewrite Eq. (10), putting in the limits of integration and simultaneously changing representation from $S_{\Phi}$ to $S_{U}$.

$$
\text { above transition: } \begin{aligned}
S_{U}(\sqrt{\mathcal{U}}) & =2 \int_{\infty}^{\mathcal{U}\left(\varphi ; \varphi_{s}\right)} d \mathcal{H}(-1 / \alpha W) g(\mathcal{H}) \\
& =2 \int_{U}^{\infty} d \mathcal{H} g(\mathcal{H}) / \sqrt{(-2 \alpha)(\mathcal{H}-\mathcal{U})} \\
\text { below transition: } S_{U}(\sqrt{-\bar{U}}) & =2 \int_{-\infty}^{\mathcal{U}\left(\varphi ; \varphi_{s}\right)} d \mathcal{H}(-1 / \alpha W) g(\mathcal{H}) \\
& =2 \int_{-\infty}^{\mathcal{U}} d \mathcal{H} g(\mathcal{H}) / \sqrt{(-2 \alpha)(\mathcal{H}-\mathcal{U})} \\
& =2 \int_{-U}^{\infty} d(-\mathcal{H}) g[-(-\mathcal{H})] / \sqrt{(2 \alpha)[(-\mathcal{H})-(-\mathcal{U})]} .
\end{aligned}
$$

In order to write $S$ in terms of coordinate $\sqrt{\mathcal{U}}$, we must use only half the signal. For a fixed value of $W$, the reduced maps $\varphi \mapsto \mathcal{U}$ and $\mathcal{U} \mapsto \varphi$ are one-to-one over a domain that does not include the fixed points. 
Both of these equations are of the correct form for application of Eq. (1), provided we use the fact (or assumption) that both $S$ and $g$ vanish outside the separatrix. We need only make the substitutions:

above transition: $x \rightarrow \sqrt{\mathcal{U}}$,

$$
\begin{aligned}
u=r^{2} & \rightarrow \mathcal{H}, \\
f(u) & \rightarrow g(\mathcal{H}) / \sqrt{-2 \alpha}, \\
g(x) & \rightarrow S_{U}(\sqrt{\mathcal{U}}) .
\end{aligned}
$$

below transition: $x \rightarrow \sqrt{-\bar{U}}$,

$$
\begin{gathered}
u=r^{2} \rightarrow-\mathcal{H}, \\
f(u) \rightarrow g(-(-\mathcal{H})) / \sqrt{2 \alpha}, \\
g(x) \rightarrow S_{U}(\sqrt{-\bar{U}}) .
\end{gathered}
$$

Reversing these in Eq. (7) then yields the result, above transition,

$$
\begin{aligned}
& \mathcal{H} g(\mathcal{H}) / \sqrt{-2} \alpha \\
& \quad=-\frac{1}{\pi} \int_{\sqrt{\mathcal{H}}}^{\infty} \frac{d(\sqrt{\mathcal{U}})}{\sqrt{1-\overline{\mathcal{H}} / \mathcal{U}}} \frac{d}{d(\sqrt{\mathcal{U}})}\left(\sqrt{\mathcal{U}} S_{U}(\sqrt{\mathcal{U}})\right) \\
& \quad=\frac{1}{\pi} \int_{\infty}^{\varphi_{1}(\mathcal{H})} \frac{d \varphi}{\sqrt{1-\overline{\mathcal{H}} / \bar{U}}} \frac{d}{d \varphi}\left(\sqrt{\mathcal{U}} S_{\Phi}(\varphi)\right),
\end{aligned}
$$

with a corresponding expression below transition. The lower limit of infinity is formal; it need extend no farther than the separatrix, since $S=0$ outside the separatrix. $\varphi=\varphi_{1}(\mathcal{H})$ is the (first) point reached at which the orbit whose Hamiltonian has value $\mathcal{H}$ intersects the $W=0$ axis: that is, $\varphi_{1}(\mathcal{H})=$ larger $\varphi$ satisfying $H(\varphi, 0)=\mathcal{H}$.

\section{A FEW COMMENTS}

Equation (11) is our principal result. It provides a linear filter from which to construct an equilibrium distribution function from a beam profile. Notice the following. (a) The value of the distribution function at the point of synchrony, $g(0)$, is left undetermined, apart from continuity:

$$
g(0)=\lim _{\mathcal{H} \rightarrow 0} g(\mathcal{H})
$$

It is a general issue in tomography.

(b) In the integrand of Eq. (11) $\mathcal{H}$ satisfies, $\mathcal{H}<\mathcal{U}(\varphi)$. This does not contradict the inequalities written on the previous page, which refer to $H$ as a function evaluated within the separatrix.

(c) In the intermediate steps I silently used the fact that $\mathcal{U}$ was convex, up or down, between the stable fixed point and the separatrix. However, in the answer's final form, that condition does not appear explicitly. Apart from convexity, $\mathcal{U}$ can be a reasonably arbitrary "potential." It is certainly not necessary to assume that it must arise from a pure sinusoidal field.

(d) Only half the signal is used. Breaking the signal into two parts, on each side of the synchronous phase, and processing them separately should give identical results. [This is not the same as claiming that $S_{\Phi}(\varphi)=S_{\Phi}(-\varphi)$, which generally will not be the case.] Doing so can serve to check (i) that we have identified $t_{s}$ or, equivalently, $\varphi_{s}$ correctly from the signal and (ii) our key assumption that the distribution is in equilibrium is valid.

(e) Another check can be done by finding the cumulative distribution as a function of action coordinate, $I$, say, $G(I)$, which is possible if the distribution is in equilibrium. When acceleration is adiabatic, $G(I)$ should not change throughout the ramp, because action is an adiabatically invariant coordinate.

(f) The square root singularity will be annoying when it comes down to numerical integration, but it can be handled. For example, it can be removed via an integration by parts. Using

$$
\frac{1}{\sqrt{1-\mathcal{H} / \mathcal{U}}}=\frac{\sqrt{\mathcal{U}}}{\sqrt{\mathcal{U}-\mathcal{H}}}=\frac{2 \sqrt{\mathcal{U}}}{d \mathcal{U} / d \varphi} \frac{d}{d \varphi}(\sqrt{\mathcal{U}-\overline{\mathcal{H}}}),
$$

we get

$$
\mathcal{H} g(\mathcal{H}) / \sqrt{-2 \alpha}=\frac{1}{\pi} \int_{\varphi_{1}(\mathcal{H})}^{\infty} d \varphi \sqrt{\mathcal{U}-\mathcal{H}} \frac{d}{d \varphi}\left[\frac{2 \sqrt{\mathcal{U}}}{d \mathcal{U} / d \varphi}\left(\frac{d}{d \varphi}[\sqrt{\mathcal{U}} S(\varphi)]\right)\right]
$$

This is effectively what would have been obtained by starting from Eq. (6) rather than Eq. (5). It removes the square root singularity but requires taking two derivatives of the signal. A better numerical trick may be to introduce subtractions and cutoffs.

$$
\begin{aligned}
\mathcal{H} g(\mathcal{H}) / \sqrt{-2 \alpha} & =\frac{1}{\pi} \int_{\infty}^{\varphi_{1}(\mathcal{H})} \frac{d \varphi}{\sqrt{1-\overline{\mathcal{H}} / \mathcal{U}}}(\cdots) \\
& =\frac{1}{\pi} \int_{\infty}^{\varphi_{1}(\mathcal{H})} \frac{d \varphi}{\sqrt{1-\overline{\mathcal{H}} / \bar{U}}}\left[(\cdots)-\left.(\cdots)\right|^{\varphi=\varphi_{1}(\mathcal{H})}\right]+\left.\frac{1}{\pi} \int_{\infty}^{\varphi_{1}(\mathcal{H})} \frac{d \varphi}{\sqrt{1-\overline{\mathcal{H}} / \mathcal{U}}}(\cdots)\right|^{\varphi=\varphi_{1}(\mathcal{H})} .
\end{aligned}
$$

The first integrand has no singularity; the second can be handled with a cutoff: 


$$
\frac{1}{\pi} \int_{\infty}^{\varphi_{1}(\mathcal{H})} \frac{d \varphi}{\sqrt{1-\mathcal{H} / \mathcal{U}}}=\left(\frac{1}{\pi} \int_{\infty}^{\varphi_{1}(\mathcal{H})-\epsilon} \frac{d \varphi}{\sqrt{1-\mathcal{H} / \bar{U}}}+\frac{1}{\pi} \int_{\epsilon}^{\varphi_{1}(\mathcal{H})} \frac{d \varphi}{\sqrt{1-\mathcal{H} / \mathcal{U}}}\right)
$$

where $\epsilon$ is chosen sufficiently small so that we can approximate $1-\mathcal{H} / \mathcal{U} \propto\left[\varphi-\varphi_{1}(\mathcal{H})\right]+O\left(\epsilon^{2}\right)$ and evaluate the last integral exactly. We reserve further discussion of numerical issues for another occasion.

[1] G. Jackson, Technical Report, Fermi National Accelerator Laboratory, FERMILAB Report No. FN0469, 1987. Available electronically at http://fnalpubs. fnal.gov/ archive/fn/FN-0469.pdf.

[2] S. Hancock, M. Lindroos, and S. Koscielniak, Phys. Rev. ST Accel. Beams 3, 124202 (2000).

[3] C. Montag, N. D'Imperio, J. Kewisch, and R. Lee, Phys. Rev. ST Accel. Beams 5, 082801 (2002).

[4] H. Schlarb, in Proceedings of the EPAC 2000, Vienna, Austria, pp. 187-191, available electronically at http://accelconf.web.cern.ch/AccelConf/e00/PAPERS/ WEXF201.pdf.

[5] M. Geitz, Ph.D. thesis, University of Hamburg, 1999 (DESY Report No. DESY-THESIS-1999-033, 1999).

[6] M. Hüning, Ph.D. thesis, University of Hamburg, 2002 (DESY Report No. DESY-THESIS-2002-029, 2002).

[7] C.-Y. Tan, Fermilab Technical Memo No. FERMILABTM-2155, 2001.

[8] N. H. Abel, Magazin for Naturvidenskaberne, Årgang I, Bind2, Christina (1823), pp. 11-27.

[9] N. H. Abel, J. Reine Angew. Math. Bd I (1826).
[10] This discovery is frequently credited to a paper written in 1826, Résolution d'un problème de mecanique [9]. Abel's 1823 paper, Solution de quelques problèmes à l'aide d'integrales définies, can be read at the web site http: // math-sahel.ujf-grenoble.fr/NUMDAM/abel.html. It appears to contain the essential pieces of his transform.

[11] H. Hochstadt, Integral Equations (Wiley-Interscience, New York, 1973).

[12] W. Pogorzelski, Integral Equations and their Applications (Pergamon, Oxford, 1966).

[13] The physics problem suggests that $\psi(t)$ should be monotonically increasing, but it is sufficient that it be continuous [11]. Physically, continuity precludes the possibility that the path possesses a roller-coaster-like dip, a requirement for the existence of the function $y(z)$.

[14] J. Radon, Ber. Verh. Sächs. Akad. Wiss. Leipzig, MathNat. 69, 262 (1917).

[15] P.W. Krempl, CERN Report No. MPS/Int. BR/74-1, 1974.

[16] J. M. Kats, in Proceedings of the 1991 Particle Accelerator Conference, San Francisco, available electronically at http://accelconf.web.cern.ch/AccelConf/p91/ PDF/PAC1991_0306.PDF.

[17] C. Carli and M. Chanel, CERN Report No. CERN/PS 2000-023, 2000. Available electronically at http://wwwlibrary.desy.de/preparch/cern/ps/ps00-023.ps.gz.

[18] Symbols, $f$ and $g$, defined in the previous section, are overloaded in this one. 